\title{
Transcranial Magnetic Stimulation to the Frontal Operculum and Supramarginal Gyrus Disrupts Planning of Outcome- Based Hand-Object Interactions
}

\author{
Eugene Tunik, ${ }^{1,2}$ On-Yee Lo, ${ }^{2}$ and Sergei V. Adamovich ${ }^{1,3}$ \\ ${ }^{1}$ Department of Rehabilitation and Movement Science, University of Medicine and Dentistry of New Jersey, Newark, New Jersey 07107 , ${ }^{2}$ Department of \\ Physical Therapy, New York University, New York, New York 10010, and ${ }^{3}$ Department of Biomedical Engineering, New Jersey Institute of Technology, \\ University Heights, Newark, New Jersey 07102
}

\begin{abstract}
Behavioral data suggest that goals inform the selection of motor commands during planning. We investigated the neural correlates that mediate planning of goal-oriented actions by asking 10 healthy subjects to prepare either a goal-specific movement toward a common object (a cup), with the intent of grasping-to-pour (liquid into it) or grasping-to-move (to another location) the object, or performing a non-object-oriented stimulus-response task (move a finger). Single-pulse transcranial magnetic stimulation was administered on $50 \%$ of trials to the supramarginal gyrus (SMG), anterior intraparietal sulcus, inferior frontal gyrus opercularis (IFGo), and triangularis during motor planning. Stimulation to SMG and IFGo caused a significant delay in planning goal-oriented actions but not responses to an arbitrary stimulus. Despite the delay, movement execution was not affected, suggesting that the motor plan remained intact. Our data implicate the SMG and IFGo in planning goal-oriented hand-object interactions.
\end{abstract}

Key words: motor control; reach-to-grasp; premotor; parietal; motor; motor intention

\section{Introduction}

Planning for how to interact with an object (hand-object interaction) is usually determined by the goal of the task (Marteniuk et al., 1987; Cohen and Rosenbaum, 2004; Johnson-Frey et al., 2005; Rosenbaum et al., 2006; Ansuini et al., 2008). The neural substrates involved in planning hand-object interactions as a function of task goal remain unknown. Here, we use transcranial magnetic stimulation (TMS) to ascribe a causal role for inferior frontal gyrus opercularis (IFGo) and the supramarginal gyrus (SMG) in planning hand-object interactions.

Neuroimaging work probing neural networks involved in goal oriented hand-object interactions reveal an inferior frontoparietal network for observation/naming of objects, tools, and hand postures (Kellenbach et al., 2003; Binkofski et al., 2004; Boronat et al., 2005; Grafton and Hamilton, 2007; Hamilton and Grafton, 2008). More recent functional MRI (fMRI) paradigms reveal repetition suppression effects for observation of action outcomes in the left anterior intraparietal sulcus (aIPS) and for observation of hand-arm trajectories/outcomes in IFG (Grafton and Hamilton, 2007; Hamilton and Grafton, 2008). A more extensive network involving the left SMG (BA40) and the IFGo is also recruited

Received 0ct. 2, 2008; revised 0ct. 31, 2008; accepted Nov. 7, 2008.

This work was supported by New York University, Steinhardt School of Culture, Education, and Human Development (E.T.), and Rehabilitation Engineering Research Center Grant H133E050011 from the National Institute on Disability and Rehabilitation Research (S.V.A.).

Correspondence should be addressed to Dr. Eugene Tunik, Department of Rehabilitation and Movement Science, University of Medicine and Dentistry of New Jersey, 65 Bergen Street, Newark, NJ 07107. E-mail: tunikeu@umdnj.edu.

DOI:10.1523/JNEUROSCI.4734-08.2008

Copyright $\odot 2008$ Society for Neuroscience $\quad$ 0270-6474/08/2814422-06\$15.00/0 when planning or pantomiming common tool-based actions (Johnson-Frey et al., 2005), and in the case of left SMG, particularly for temporally removed compared with more immediate goals (Majdandzić et al., 2007). Consistent with these observations, inferior parietal lobule lesions (and/or disrupted connections to premotor and subcortical structures) often result in ideomotor apraxia, or impaired production (Sirigu et al., 1995; Hanna-Pladdy et al., 2001; Buxbaum et al., 2003, 2005b, 2007) and imagery (Ochipa et al., 1997) of meaningful gestures or hand-object interactions. Notably, it has been reported that lesions circumscribing BA40 impair selection of comfortable hand-arm configurations for power- and pincer-grasp (Johnson et al., 2002; Buxbaum et al., 2005a). Thus, IFGo and SMG may be important for planning goal-oriented hand-object interactions.

To investigate this, we asked subjects to reach-to-grasp a cup placed upside-down. We randomly varied the hand-object interaction requirement by instructing different goals: grasping the cup to pour liquid into it (thumb-down grasp) versus grasping the cup to move it over (over-the-top grasp). We interleaved two additional control instructions: a lift-the-finger cue that did not require acting on an object (to rule out arbitrary stimulusresponse mapping effects) and a rest cue to discourage precued movements. The additional conditions also increased demands on trial-to-trial replanning. TMS was administered (50\% of trials) immediately after the cue to SMG, IFGo, and two control sites: aIPS and IFG triangularis (IFGt). Recent data suggest that perimovement, but not premovement, TMS to aIPS disrupts actions, implicating aIPS in guiding rather than planning actions (Rice et al., 2006) and that IFGt processes language (Amunts et 


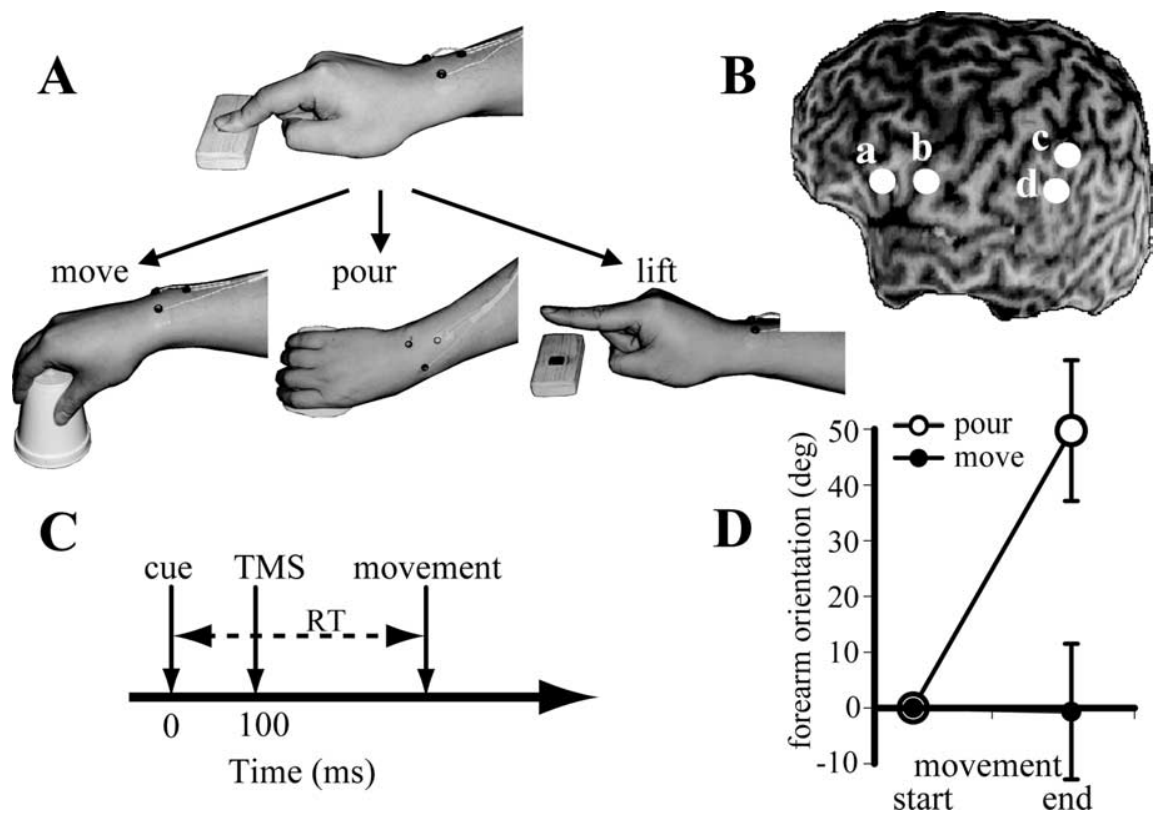

Figure 1. A, Instructions and corresponding responses in the motor conditions. $\boldsymbol{B}$, Three-dimensional rendering of one subject's structural MRI with marked cortical sites: $a$, IFGt; $b$, IFGo; $c$, alPS; $d$, SMG. C, Time frame of events. $\boldsymbol{D}$, Group means \pm 1 SD of the initial and final forearm orientation angle for the task conditions.

al., 1999). The spatial proximity to SMG and IFGo also make these robust control sites. We predicted that TMS to SMG and IFGo would impair planning (prolong reaction time) for goaloriented actions but not for arbitrary stimulus-response mappings.

\section{Materials and Methods}

Subjects. Ten right-handed (Oldfield, 1971) healthy subjects (7F; mean age $\pm 1 \mathrm{SD}, 26.3 \pm 3.27$ years old) participated after providing informed consent.

Setup and procedure. Seated subjects pressed a start button using their right index finger while awaiting an instruction cue displayed on a monitor placed $45 \mathrm{~cm}$ away. The instruction (duration, $500 \mathrm{~ms}$ ) cued subjects to do one of the following as fast as possible (Fig. 1A). Task (40 trials): reach-to-grasp a cup (placed upside-down $20 \mathrm{~cm}$ away) and turn it over as if to pour liquid into it (pour) or move it over three centimeters to the right (move); control (20 trials): lift your finger off the button for two seconds (lift), which required responding to the cue without acting on an object-oriented goal; rest (10 trials): maintain your finger on the button. Rest trials were not analyzed but assured that subjects remained attentive and increased the need to replan from trial to trial. The intertrial interval was $5 \mathrm{~s}$.

TMS. Single-pulse TMS (MAGSTIM Rapid ${ }^{2}$, double-70 mm coil) was administered on $50 \%$ of the trials $100 \mathrm{~ms}$ after cue onset, consistent with the likely timing of information processing in the regions of interest (Schluter et al., 1998, 1999). Four left-hemisphere sites were stimulated (Fig. 1B): (1) inferior bank of the anterior intraparietal sulcus at its junction with the postcentral sulcus (aIPS); (2) SMG; (3) inferior frontal operculum (IFGo); (4) inferior frontal gyrus pars triangularis (IFGt). The spatial proximity between IFGo-IFGt and aIPS-SMG controlled for nonspecific effects. Frameless stereotaxic neuronavigation (Brainsight Frameless, Rogue Research) was used to mark the TMS sites of interest on each subject's structural MRI, to localize the coil position, and monitor its position throughout the TMS session. The experimenter held the TMS coil with the handle tangential to the surface of the skull and perpendicular to the gyrus (Brasil-Neto et al., 1992) (parietal sites: handle downward; frontal sites: handle backward). Motor threshold was determined as the intensity producing a visible contraction of the intrinsic hand muscles on $50 \%$ of 10 consecutive trials with the coil over the hand area of M1. We used $110 \%$ of this intensity for the experiment. Participants completed a side-effects questionnaire (Machii et al., 2006) before and after the experiment. Each cortical site was tested in a separate block. Within-block trial order was randomized. Block order was counterbalanced across subjects.

Dependent measures. Kinematic data were obtained by localizing the three-dimensional position of three infrared light emitting diodes (Optotrak Certus, Northern Digital, $100 \mathrm{~Hz}$ ) attached to the radius dorsal tubercle, ulnar styloid (referred to as the wrist), and between these markers $3 \mathrm{~cm}$ proximally (Fig. $1 \mathrm{~A}$ ). Offline, missing samples were interpolated and the data were low-pass filtered $(10 \mathrm{~Hz})$, and analyzed using custom-written Matlab (MathWorks) software. For the two task conditions, we defined movement onset and offset as the time at which the sagittal velocity of the wrist exceeded or fell below, respectively, $5 \%$ of the peak sagittal velocity for at least $50 \mathrm{~ms}$.

We used reaction time (RT) to quantify the time needed to plan an action. In past experiences with similar choice RT paradigms, we found subjects sometimes used a strategy in which they pause between button release and movement onset, affording more time to make a plan. Thus, we quantified the RT as the interval between the presentation of the cue (move, pour) and movement onset toward the object. The control condition did not involve a reach, so RT was defined as the interval between the cue and button release. For comparisons between the task and control conditions, the percentage change in the RT (\%RT) was calculated as $\left(\left(\mathrm{RT}_{\mathrm{TMS}}-\mathrm{RT}_{\mathrm{NoTMS}}\right) / \mathrm{RT}_{\mathrm{NoTMS}}\right)^{*} 100$. Measures to quantify task execution were: movement time, the interval between movement onset and offset; initial and final forearm orientation, the angle between a vertical line and the normal to a plane defined by the three markers, $250 \mathrm{~ms}$ before the instruction cue appeared and at the time of movement offset; variability in the final position of the wrist, the volume of the $95 \%$ confidence ellipsoid whose axes are directed along the three principal eigenvectors of the covariance matrix that contains coordinates of the final wrist position and the yaw and pitch angles of the first eigenvector (Adamovich et al., 2001); peak angular acceleration and velocity, time to peak angular acceleration and velocity, peak sagittal acceleration and velocity, time to peak sagittal acceleration and velocity of the wrist.

Statistical analysis. Variables were analyzed with a three-way repeatedmeasures ANOVA, factors (levels): brain-site (aIPS, SMG, IFGo, IFGt), TMS condition (TMS, no-TMS), and task (move, pour). We performed a separate two-way ANOVA (brain-site, TMS condition) for RT in the control condition since button release defined movement onset in this condition. All RTs were also compared in a single ANOVA as \%RT [brain-site (aIPS, SMG, IFGo, IFGt), motor condition (move, pour, control)]. Significant effects were explored with two-way ANOVAs and Student-Newman-Keuls post hoc tests. Significance was set at 0.05 .

\section{Results}

\section{Initial hand orientation}

Subjects were asked to maintain a consistent initial hand orientation. Figure $1 D$ shows the average hand orientation before cue presentation ranged from $-0.00007^{\circ}\left( \pm 0.004^{\circ}\right)$ to $0.003^{\circ}$ $\left( \pm 0.012^{\circ}\right)$ across brain-site, TMS, and task conditions (all main effects and interactions, $p>0.05$ ), suggesting that any effects could not be attributed to differences in initial calibration.

\section{Task planning}

Figure $2 \mathrm{~A}$ shows each subject's mean RT (open circles) in the pour and move conditions for each brain-site and TMS condition. The horizontal bars overlaying the individual subject data represent group means $\pm 1 \mathrm{SD}$. The ANOVA for RT revealed a 

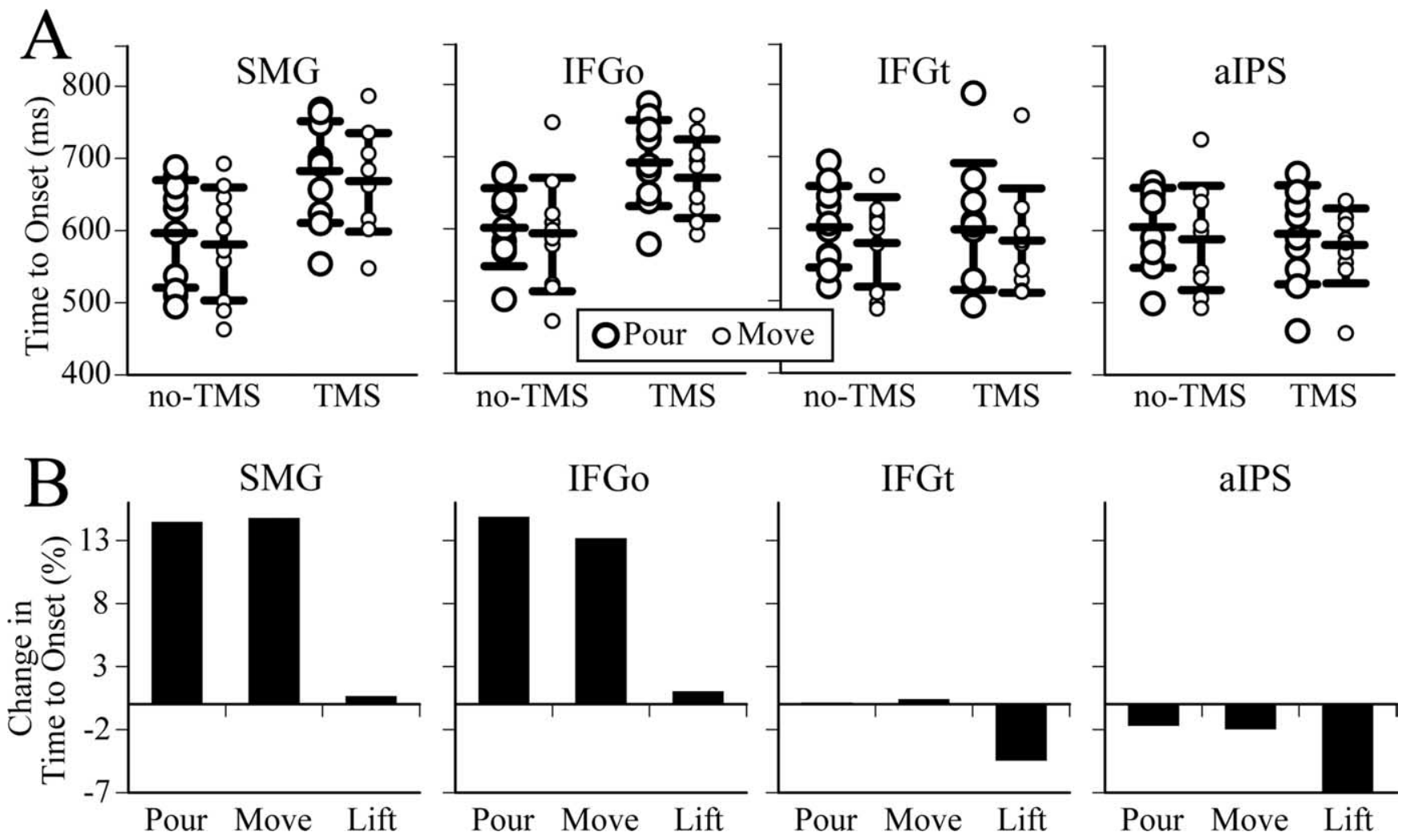

Figure 2. A, Each subject's (circles) and group (bars) mean \pm 1 SD RT in the pour and move conditions for the different TMS and brain-site conditions. $\boldsymbol{B}$, The RT shown as a percentage change in the TMS relative to no-TMS condition in the pour, move, and lift conditions.

significant main effect of brain-site $\left(F_{(3,27)}=8.6, p=0.0004\right)$, TMS condition $\left(F_{(1,9)}=26.5, p=0.0006\right)$, and a significant brain-site $\times$ TMS condition interaction $\left(F_{(3,27)}=16.6, p<\right.$ 0.0001 ). Since we did not observe any main effect of task (move vs pour), we further analyzed the RT data by collapsing across task conditions. The two-way ANOVA revealed a main effect of brainsite $\left(F_{(3,57)}=16.1, p<0.0001\right)$, TMS condition $\left(F_{(1,19)}=44.2\right.$, $p<0.0001)$, and a brain-site $\times$ TMS condition interaction $\left(F_{(3,57)}=24.6, p<0.0001\right)$. Post hoc tests showed that the effects were driven by significant differences between the TMS and noTMS conditions for SMG and IFGo but not the other sites.

To determine if TMS-induced delays in planning were general in nature (stimulus-response mapping problem) or specific to object-oriented grasping, we analyzed RT in a simple stimulusresponse task when no interaction with the object was required. No significant delay in planning was noted between the no-TMS and TMS conditions at all four brain sites [mean \pm 1SD: SMG $370.8( \pm 54.9)$ vs $372.9( \pm 39.3)$; IFGo: $362.1( \pm 42.4)$ vs 365.6 ( \pm 41.8$)$; IFGt: $387.7( \pm 63.7)$ vs 370.7 ( \pm 48.8$)$; aIPS: 386.9 $( \pm 64.5)$ vs 360.9 ( \pm 40.3$)$; main effects/interactions, $p>0.2$ ].

To further test for TMS effects on planning, we compared RT in the task (collapsed across pour and move) and control conditions as a percentage change in the TMS relative to no-TMS conditions in a two-way ANOVA (Fig. $2 B$ ). We noted a significant main effect for motor condition $\left(F_{(1,9)}=8.8, p=0.02\right)$ and brain-site $\left(F_{(3,27)}=7.3, p<0.001\right)$. Post hoc analysis revealed that the \%RT was significantly higher in the task condition than in the control condition when TMS was administered to SMG $(15.2 \% \pm 7.9 \%$ vs $2.2 \pm 16 \%)$ and IFGo $(14.6 \pm 8 \%$ vs $1.9 \% \pm 15.1 \%)$.

\section{Task execution}

To determine whether the delays in planning led to differences in the reaching movement, we analyzed several reach-related kinematic measures. Figure $3 A$ shows the mean three-dimensional trajectory profiles of the wrist movement in each condition for a representative subject. The variability in the final position of the wrist is displayed as a $95 \%$ confidence ellipsoid. No main or interaction effects were observed for the volume and orientation (yaw and pitch) of the first eigenvector of the confidence ellipsoids. Moreover, no significant effects were observed for the peak sagittal acceleration and velocity and for the time to peak sagittal acceleration and velocity of the wrist, suggesting that early reach kinematics were unaffected across task and TMS conditions.

Since each task required a unique final forearm orientation, we also analyzed the forearm rotation angle. Figure $1 D$ shows the group mean $\pm 1 \mathrm{SD}$ joint angle for the grasp-to-pour and graspto-move tasks. In the grasp-to-pour condition, subjects used a thumb-down posture, rotating their hand an average $50.4^{\circ}$ more than they had for the grasp-to-move task (task main effect: $F_{(1,9)}$ $=123.7, p<0.0001)$. The greater forearm rotation in the graspto-pour condition was associated with a longer movement time relative to the grasp-to-move condition (mean difference between conditions, $72.2 \mathrm{~ms}$; task main effect: $F_{(1,9)}=19.2, p<$ $0.002)$. No other main effects or interactions were noted suggesting that the final forearm orientation was unaffected by TMS.

Last, to determine if TMS affected forearm orientation early in the movement, we analyzed the time traces of the forearm rotation angle. Figure $3 B$ shows the group mean $\pm 1 \mathrm{SD}$ trajectories of the forearm rotation for each task and TMS condition. The angle trajectories of grasp-to-pour trials remained invariant in shape 


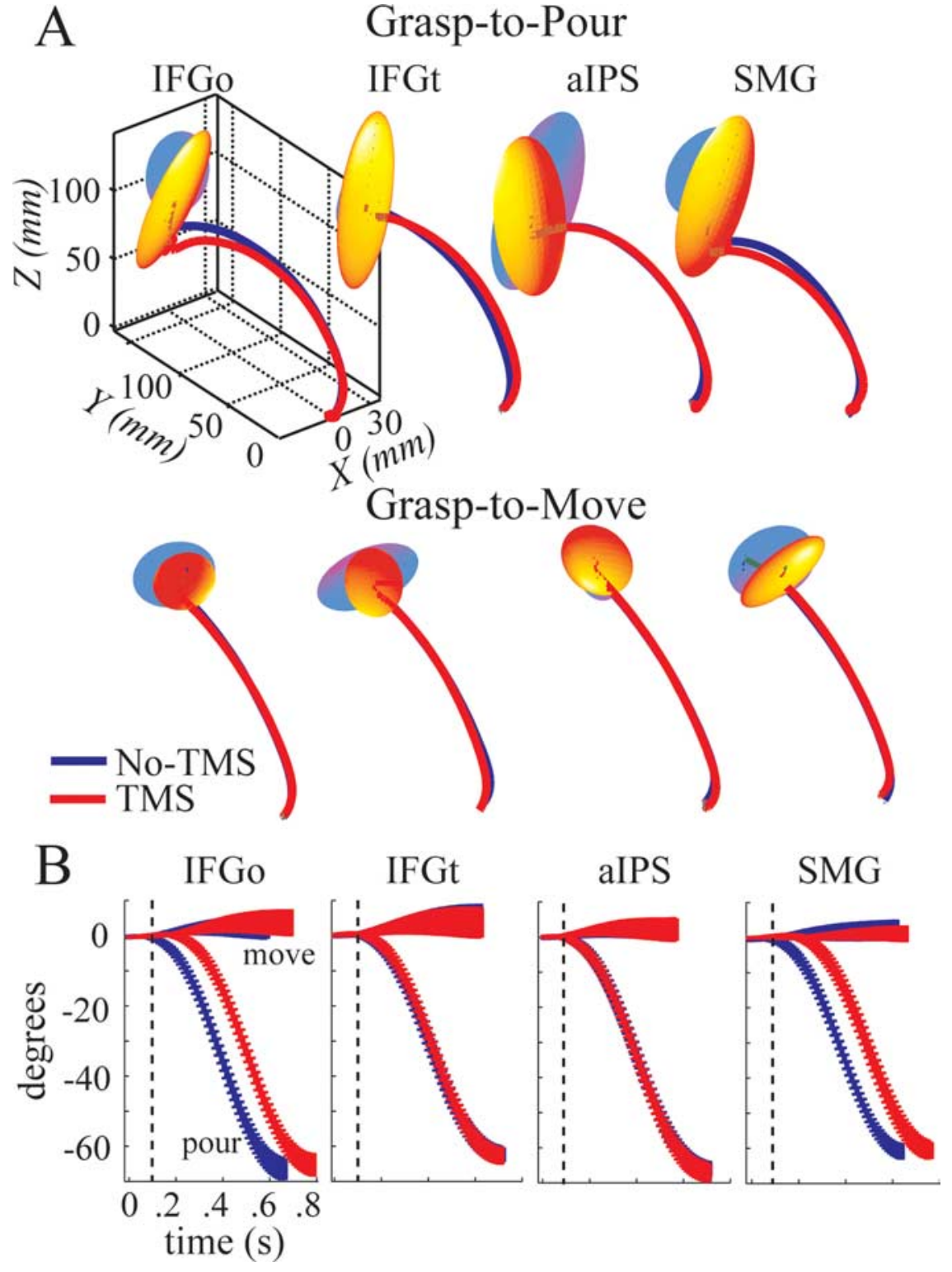

Figure 3. A, A representative subject's mean hand trajectory profiles. Variations in the final wrist position around the mean are shown by $95 \%$ confidence ellipsoids (see Materials and Methods). $\boldsymbol{B}$, Group mean \pm 1 SD orientation angle of the forearm for each brain-site, task, and TMS condition. Time of TMS pulse is shown by dashed line.

between TMS conditions but were delayed when IFGo and SMG were stimulated. To quantify the invariance in the early forearm rotation across TMS conditions, we analyzed the peak angular acceleration and velocity and the time to peak angular velocity and acceleration. Peak angular acceleration and velocity were affected by task $\left(F_{(1,9)}=322.4, p<0.0001\right.$ and $F_{(1,9)}=317.6, p<$ 0.0001 ) with the forearm rotation being faster in the pour condition. No significant main effects of TMS or brain-site, or interactions, were noted. These findings suggest that (1) the two goals were achieved using distinct coordination patterns, and (2) TMS did not affect movement execution.

\section{Discussion}

The role of a ventral frontoparietal network in planning and guiding goal-oriented action

Goals influence our interactions with objects. For example, individuals grasp a dowel at distinct positions along its height when planning to place it onto shelves of different height (Cohen and
Rosenbaum 2004; Rosenbaum et al., 2006) and reach-to-grasp kinematics for objects vary as a function of how to use the object (Marteniuk et al., 1987; Johnson-Frey et al., 2005; Ansuini et al., 2008) or the size/ location of the surface onto which an object will be placed (Gentilucci et al., 1997; Ansuini et al., 2006). Our study investigated the neural mechanisms that allow goals to influence actions. Conceptually, acting on any object can carry an implicit goal, even if that goal is not made explicit to the subject. Since goals may be inadvertently linked to objects, it presents a challenge to contrast "goal-" with "non-goal-" oriented actions. We avoided this hurdle by having subjects act on an object in response to different goals and contrasted this with a non-object-oriented action. Our data demonstrate that TMS to SMG and IFGo during the planning phase delayed the onset of a response (action planning) for object-oriented action but not for arbitrary stimulus-response mappings. Despite delaying planning, TMS did not affect any spatiotemporal parameters of movement execution suggesting that the action plan remained intact, albeit delayed. The lack of TMS-induced effects in aIPS in our study are consistent with our previous data showing that TMS to aIPS disrupts actions only if the pulse is delivered during execution (not planning) of grasp (Tunik et al., 2005; Rice et al., 2006), suggesting that the primary role of aIPS seems to be of guidance rather than planning of actions.

In the monkey, the homolog of human SMG corresponds to BA7b (Brodmann, 1909), or area PF (Von Economo, 1929) (for review, see Rizzolatti and Matelli, 2003). Retrograde tracer injections into BA7b labels neurons in sensorimotor cortices, polymodal cortical areas, aIPS, and IFGo [Lewis and Van Essen (2000), their Fig. 7]. Neuroimaging tractography studies suggest that a homologous corticocortical network exists in humans (Rushworth et al., 2006). In the backdrop of our previous data, that perimovement, but not premovement, TMS to aIPS disrupts kinematics (Tunik et al., 2005; Rice et al., 2006), we argue that SMG and aIPS may have complementary roles in planning and guiding, respectively, reach-to-grasp actions. Through their mutual connections with the ventral premotor cortex, SMG may be involved in goal-oriented formation of plans and selection of actions. Conversely, aIPS may be dynamically involved in monitoring the fit between hand-object interactions and the intended outcome of the action.

\section{Planning for temporally removed goals or responding to a stimulus?}

Our paradigm resembled a choice reaction time task in that each instruction cue beckoned a unique motor response. However, our paradigm departed from traditional stimulus-response ap- 
proaches in that one set of choice conditions required selecting a goal-oriented action (move vs pour) while the other (control) condition did not. TMS to some cortical regions, such as the dorsal premotor cortex and motor cortex, can delay stimulusresponse mapping responses in simple- and choice-reaction time tasks (Schluter et al., 1998, 1999; O'Shea et al., 2007). However, it is unlikely that this explains our data since TMS-induced effects were specific to actions oriented toward an object with a goal (grasp-to-pour and grasp-to-move) and were absent in the control condition. In other words, if IFGo and SMG were simply involved in stimulus response mapping, we would expect TMS to prolong reaction times in the control condition as well, which was not the case. Thus, our data suggest that IFGo and SMG may plan actions of the hand for purposefully using an object. However, our data cannot dissociate whether information processing within IFGo and SMG is specific to the presence of an object or not. This dissociation, between objects and goals, for SMG and IFGo is currently under investigation.

\section{Contributions of IFGo and SMG to action planning}

Neurophysiological unit recordings in the anterior region of the macaque left inferior parietal lobule show that the firing rate of a substantial number of neurons is tuned for action goals rather than the kinematics of the action (Fogassi et al., 2005). The unit discharge generally begins before movement onset [Fogassi et al. (2005), their Figs. 1, 2] suggesting a role of these neurons in goal-specific motor planning. Other neurons have been shown to be tuned for different forms of grasp (i.e., conical, pincer, power, etc.) (Taira et al., 1990; Sakata et al., 1992, 1995; Murata et al., 2000). These studies suggest that disruption of a unique neural population should impair planning for the specific goal or specific grasp that a given neural population represents. However, neurons specific to different goals are intermingled among each other, and similarly, neurons specific to different grasp are intermingled as well. Given that TMS nonselectively effects an entire neural population, we did not see distinct delays in reaction time between the grasp-to-pour and grasp-to-move conditions.

A recent fMRI study addressed a similar issue (Króliczak et al., 2008). In an elegant repetition suppression design, subjects reached-to-grasp a three-dimensional object. On a trial-to-trial basis, either the object shape or the grasp kinematics were repeated or changed. The authors noted repetition suppression effects in the bilateral aIPS, left SMG, and right mid superior parietal lobe for repeated grasps and repeated objects. In contrast, repetition suppression effects in the left IFGo and bilateral dorsal premotor cortex were evident only for repeated grasps. Our study is consistent with these data in that IFGo and SMG are critical for goal-oriented actions. We extend these findings by localizing the contributions of IFGo and SMG to the planning phase of a goaldirected action. Our future TMS work is designed to understand the unique contribution of each region.

\section{References}

Adamovich SV, Archambault PS, Ghafouri M, Levin MF, Poizner H, Feldman AG (2001) Hand trajectory invariance in reaching movements involving the trunk. Exp Brain Res 138:288-303.

Amunts K, Schleicher A, Bürgel U, Mohlberg H, Uylings HB, Zilles K (1999) Broca's region revisited: cytoarchitecture and intersubject variability. J Comp Neurol 412:319-341.

Ansuini C, Santello M, Massaccesi S, Castiello U (2006) Effects of end-goal on hand shaping. J Neurophysiol 95:2456-2465.

Ansuini C, Giosa L, Turella L, Altoè G, Castiello U (2008) An object for an action, the same object for other actions: effects on hand shaping. Exp Brain Res 185:111-119.
Binkofski F, Buccino G, Zilles K, Fink GR (2004) Supramodal representation of objects and actions in the human inferior temporal and ventral premotor cortex. Cortex 40:159-161.

Boronat CB, Buxbaum LJ, Coslett HB, Tang K, Saffran EM, Kimberg DY, Detre JA (2005) Distinctions between manipulation and function knowledge of objects: evidence from functional magnetic resonance imaging. Brain Res Cogn Brain Res 23:361-373.

Brasil-Neto JP, Cohen LG, Panizza M, Nilsson J, Roth BJ, Hallett M (1992) Optimal focal transcranial magnetic activation of the human motor cortex: effects of coil orientation, shape of the induced current pulse, and stimulus intensity. J Clin Neurophysiol 9:132-136.

Brodmann K (1909) Vergleichende Lokalisationslehre der Grosshirnrinde in ihren Prinzipien Dargerstellt auf Grund des Zellenbaues. Leipzig: Barth.

Buxbaum LJ, Sirigu A, Schwartz MF, Klatzky R (2003) Cognitive representations of hand posture in ideomotor apraxia. Neuropsychologia 41:1091-1113.

Buxbaum LJ, Johnson-Frey SH, Bartlett-Williams M (2005a) Deficient internal models for planning hand-object interactions in apraxia. Neuropsychologia 43:917-929.

Buxbaum LJ, Kyle KM, Menon R (2005b) On beyond mirror neurons: internal representations subserving imitation and recognition of skilled object-related actions in humans. Brain Res Cogn Brain Res 25:226-239.

Buxbaum LJ, Kyle K, Grossman M, Coslett HB (2007) Left inferior parietal representations for skilled hand-object interactions: evidence from stroke and corticobasal degeneration. Cortex 43:411-423.

Cohen RG, Rosenbaum DA (2004) Where grasps are made reveals how grasps are planned: generation and recall of motor plans. Exp Brain Res 157:486-495.

Fogassi L, Ferrari PF, Gesierich B, Rozzi S, Chersi F, Rizzolatti G (2005) Parietal lobe: from action organization to intention understanding. Science 308:662-667.

Gentilucci M, Negrotti A, Gangitano M (1997) Planning an action. Exp Brain Res 115:116-128.

Grafton ST, Hamilton AF (2007) Evidence for a distributed hierarchy of action representation in the brain. Hum Mov Sci 26:590-616.

Hamilton AF, Grafton ST (2008) Action outcomes are represented in human inferior frontoparietal cortex. Cereb Cortex 18:1160-1168.

Hanna-Pladdy B, Heilman KM, Foundas AL (2001) Cortical and subcortical contributions to ideomotor apraxia: analysis of task demands and error types. Brain 124:2513-2527.

Johnson SH, Sprehn G, Saykin AJ (2002) Intact motor imagery in chronic upper limb hemiplegics: evidence for activity-independent action representations. J Cogn Neurosci 14:841-852.

Johnson-Frey SH, Newman-Norlund R, Grafton ST (2005) A distributed left hemisphere network active during planning of everyday tool use skills. Cereb Cortex 15:681-695.

Kellenbach ML, Brett M, Patterson K (2003) Actions speak louder than functions: the importance of manipulability and action in tool representation. J Cogn Neurosci 15:30-46.

Króliczak G, McAdam TD, Quinlan DJ, Culham JC (2008) The human dorsal stream adapts to real actions and 3D shape processing: a functional magnetic resonance imaging study. J Neurophysiol 100:2627-2639.

Lewis JW, Van Essen DC (2000) Corticocortical connections of visual, sensorimotor, and multimodal processing areas in the parietal lobe of the macaque monkey. J Comp Neurol 428:112-137.

Machii K, Cohen D, Ramos-Estebanez C, Pascual-Leone A (2006) Safety of rTMS to non-motor cortical areas in healthy participants and patients. Clin Neurophysiol 117:455-471.

Majdandzić J, Grol MJ, van Schie HT, Verhagen L, Toni I, Bekkering H (2007) The role of immediate and final goals in action planning: an fMRI study. Neuroimage 37:589-598.

Marteniuk RG, MacKenzie CL, Jeannerod M, Athenes S, Dugas C (1987) Constraints on human arm movement trajectories. Can J Psychol 41:365-378.

Murata A, Gallese V, Luppino G, Kaseda M, Sakata H (2000) Selectivity for the shape, size, and orientation of objects for grasping in neurons of monkey parietal area AIP. J Neurophysiol 83:2580-2601.

Ochipa C, Rapcsak SZ, Maher LM, Rothi LJ, Bowers D, Heilman KM (1997) Selective deficit of praxis imagery in ideomotor apraxia. Neurology 49:474-480. 
Oldfield RC (1971) The assessment and analysis of handedness: the Edinburgh inventory. Neuropsychologia 9:97-113.

O'Shea J, Sebastian C, Boorman ED, Johansen-Berg H, Rushworth MF (2007) Functional specificity of human premotor-motor cortical interactions during action selection. Eur J Neurosci 26:2085-2095.

Rice NJ, Tunik E, Grafton ST (2006) The anterior intraparietal sulcus mediates grasp execution, independent of requirement to update: new insights from transcranial magnetic stimulation. J Neurosci 26:8176-8182.

Rizzolatti G, Matelli M (2003) Two different streams form the dorsal visual system: anatomy and functions. Exp Brain Res 153:146-157.

Rosenbaum DA, Halloran ES, Cohen RG (2006) Grasping movement plans. Psychon Bull Rev 13:918-922.

Rushworth MF, Behrens TE, Johansen-Berg H (2006) Connection patterns distinguish 3 regions of human parietal cortex. Cereb Cortex 16:1418-1430.

Sakata H, Taira M, Mine S, Murata A (1992) Hand-movement related neurons of the posterior parietal cortex of the monkey: their role in visual guidance of hand movements. In: Control of arm movement in space: neurophysiological and computational approaches (Camaniti R, Johnson PB, Burnod Y, eds), pp 185-198. Berlin: Springer.

Sakata H, Taira M, Murata A, Mine S (1995) Neural mechanisms of visual guidance of hand action in the parietal cortex of the monkey. Cereb Cortex 5:429-438.

Schluter ND, Rushworth MF, Passingham RE, Mills KR (1998) Temporary interference in human lateral premotor cortex suggests dominance for the selection of movements. A study using transcranial magnetic stimulation. Brain 121:785-799.

Schluter ND, Rushworth MF, Mills KR, Passingham RE (1999) Signal-, set-, and movement-related activity in the human premotor cortex. Neuropsychologia 37:233-243.

Sirigu A, Cohen L, Duhamel JR, Pillon B, Dubois B, Agid Y (1995) A selective impairment of hand posture for object utilization in apraxia. Cortex 31:41-55.

Taira M, Mine S, Georgopoulos AP, Murata A, Sakata H (1990) Parietal cortex neurons of the monkey related to the visual guidance of hand movement. Exp Brain Res 83:29-36.

Tunik E, Frey SH, Grafton ST (2005) Virtual lesions of the anterior intraparietal area disrupt goal-dependent on-line adjustments of grasp. Nat Neurosci 8:505-511.

Von Economo C (1929) The cytoarchitectonics of the human cerebral cortex. London: Oxford UP. 